\title{
MODIFICATION OF COMPOSITE MATERIAL FILLERS BY ATMOSPHERIC PLASMA DISCHARGE
}

\author{
David Tichy ${ }^{a, *}$, Pavlina Hajkova ${ }^{b}$ \\ a Technical University of Liberec, Department of Material Science, Studentska 2, 46117 Liberec, Czech Republic \\ ${ }^{b}$ Research Institute of Inorganic Chemistry, Revolucni 84, 40001 Usti nad Labem, Czech Republic \\ * corresponding author: david.tichy@tul.cz
}

\begin{abstract}
This work is focused on the observation of the influence of cold atmospheric dielectric barrier discharge (DBD) on a modification of textile samples. The main objective of the experiment is to research wettability change of textiles modified by different exposure times and also the observation of the influence of a modification ageing effect. An ambient air was used as a working gas for DBD plasma. The wettability evaluation was carried out by a drop method, in which an imprint of the drop was observed on the textile surface during various time intervals. An ageing effect of the modification was monitored within an interval of 28 days. Considerable increase of wettability of all modified samples has been proved. A fibre surface analysis was carried out by means of SEM.
\end{abstract}

KEYWORDS: atmospheric plasma, dielectric barrier discharge, glass textiles, basalt textiles, wettability.

\section{INTRODUCTION}

The necessity of an adhesion increasing among the fibres and matrix in the composite materials was known from the time when the glass fibres started to be used for composite material reinforcement. The adhesion among the fibres and the composite matrix is fundamental for transfer of stress from the matrix to the reinforcing fibres. The surface finishing techniques should influence only chemical and physical properties of the material surface layer without any changes of bulk material properties of the fibres [12]. The main motivation for the application of the surface plasma modifications of fibres used as composite reinforcement is a replacement of wet chemical methods of the surface finishing by new methods, which are friendly to the environment [3, 7]. The modification of fibre upper atomic layers with a minimum thermal and chemical degradation of the remaining part of the fibre is the main advantage of plasma modification in comparison with the traditional methods. The applications of atmospheric dielectric barrier discharge reactors as a substitution of expensive vacuum devices were introduced, when they started to provide similar results as low-pressure devices [1]. It means that the composite material fillers can also be modified by the atmospheric plasma in order to improve their performance. The improvement of the composite mechanical properties correlates with an improvement of the wettability (hydrophilicity) in the matrix-fibre system of the reinforcement. The wettability definitely influences the interfacial adhesion of the fibres and matrix thanks to the surface energy changes. The soaking effect of a coloured drop of water had been proposed and agreed as a test method for determination of the level of wettability 9. A study of ageing effect on wettability within selected conditions was the main objective of the experiments described in this article.

\section{AIMS}

The main objective of this work is to induce the chemical reactions on the surface of selected textiles by means of application of a low-temperature plasma from atmospheric dielectric barrier discharge (DBD) in the filamentary mode and a subsequent research of wettability changes after various periods of exposure as well as influence of ageing of the modified samples. The evaluation of the textile sample wettability was carried out by a drop liquid test and by an observation of the imprint of the soaking drop during various time intervals.

\section{MATERIALS AND METHODS}

\subsection{TeXtile SAMPLES}

The modified samples were made from a commercial E-glass. The textiles with plain weave were cut into the test samples with the size $50 \times 50 \mathrm{~mm}$ and thickness about $0.5 \mathrm{~mm}$.

\subsection{EXPERIMENT ALIGNMENT}

The plasma reactor (Fig. 1) consists of two plane parallel electrodes made from $\mathrm{Ag} 80 \mathrm{Cu}$ alloy covered by a $1 \mathrm{~mm}$ layer of dielectric (corundum and rubber). Both electrodes have a rectangular shape with the dimension $60 \times 50 \mathrm{~mm}$ and thickness of $8 \mathrm{~mm}$ without active cooling. The distance between the electrodes was $5 \mathrm{~mm}$ for all experiments. The discharge is ignited by means of an AC power source and fills the entire space between the electrodes. This means that the samples are completely immersed in the plasma.

Plasma treatment conditions and parameters:

- $\mathrm{AC}$ source voltage $=20 \mathrm{kV}$

- $\mathrm{AC}$ source freqeuncy $=10 \mathrm{kHz}$

- nominal power $=120 \mathrm{~W}$ 


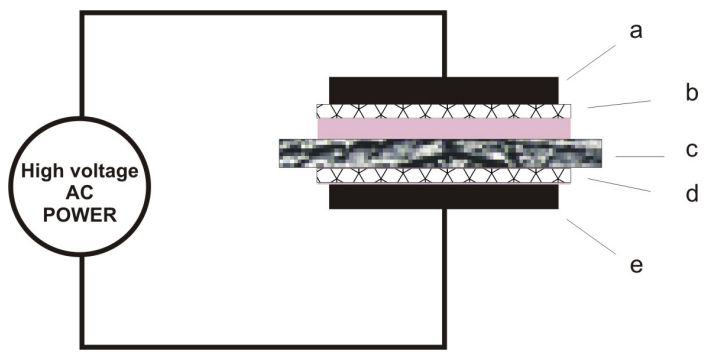

Figure 1. DBD Plasma reactor with parallel electrodes alignment; a) powered electrode, b) dielectric barrier 1 - corundum, c) textile sample, d) dielectric barrier 2 - rubber, e) grounded electrode.

- distance of electrodes $=5 \mathrm{~mm}$

- modification time periods $=1,5,10 \mathrm{~s}$

- working gas: ambient air

The evaluation of the textile sample wettability was carried out by the drop liquid test and by an observation of the area $S\left(\mathrm{~mm}^{2}\right)$ of the soaking drop during various time intervals by means of [4]. The effect of the modification ageing was monitored and tabulated during the following 28 days after the experiment was finished. All experiments were executed at room temperature, about $22^{\circ} \mathrm{C}$, dark and relative humidity of about $40 \%$.

The method presented by Pichal [5, 6] in his work was used for the determination of a modified textile wettability change. An aqueous solution of potassium permanganate with concentration of $0.125 \%$ was taken by micro-pipette. Drop volume was $20 \mu l$. The drop was placed in the centre of the textile sample and the drop behaviour was recorded by a recording device (CCD camera with HD resolution) during defined time intervals. The brand new piece of textile sample was used for each drop test.

The drop imprint was recorded during the initial $20 \mathrm{~s}$ in 1 -second intervals, then up to $30 \mathrm{~s}$ in 5 -second intervals and finally up to $60 \mathrm{~s}$ in 10 -second intervals. The first $20 \mathrm{~s}$ with 1 -second interval was selected because of very rapid soaking of the drop at the beginning of the test. The drop imprint has a circular shape so it is possible to calculate and approximate the marked area easily. The process of the drop absorption into the textile sample is clearly visible from the diagrams that have been prepared on the basis of obtained results. The textile without a plasma treatment was used as a comparative sample.

The fact that the wettability of the textile made from E-glass increased during ageing thanks to the subsequent reactions on the sample fibre surface is a very interesting result of the test. Another samples of basalt textile showed similar properties after the DBD plasma modification.

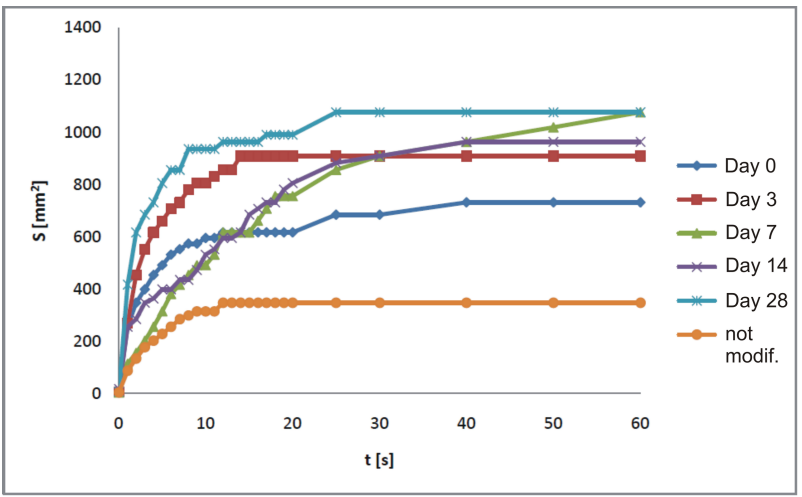

Figure 2. E-glass textile modified sample, wettability expressed by a print area, modification time $1 \mathrm{~s}$.

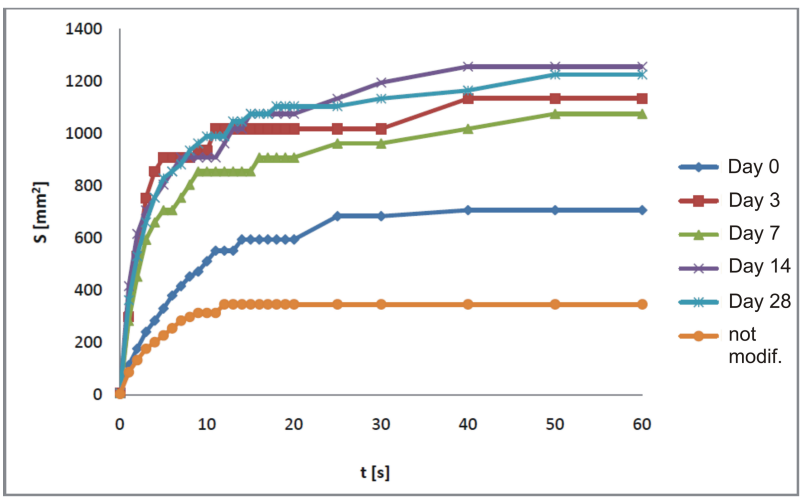

Figure 3. E-glass textile modified sample, wettability expressed by a print area, modification time $5 \mathrm{~s}$.

\section{Results AND Discussion}

\subsection{Ageing efFect of Wettability CHANGES}

Atmospheric plasma can treat various materials even those which are low temperature degradable. If the surface activation remains stable over a quite long period: treated substrates can thus be stored [10]. For this purpose the ageing effect on the samples wettability was studied in this work.

Comparing to literature it can be traced that both the enhancement of the modification effect and the recovery to the initial properties occurs during ageing. However, the reported experiments have very different treatment conditions and also various types of plasma are used [8, 3, 11, 2]. Most of the experiments are performed with an atmospheric pressure plasma jet or low-pressure devices applying a low $13.56 \mathrm{MHz}$ radio frequency power. Also, different gas compositions are used. In cases where DBD atmospheric plasma was used (either parallel or coplanar alignment), the power delivered to the plasma reactor was significantly lower than in experiments described in this work. To the best knowledge of authors, no works with similar or comparable conditions considering volume DBD plasma reactor operated at $100 \mathrm{~W}$ or more at low frequency in resonant mode are described in literature.

In this case of plasma DBD modification of tex- 


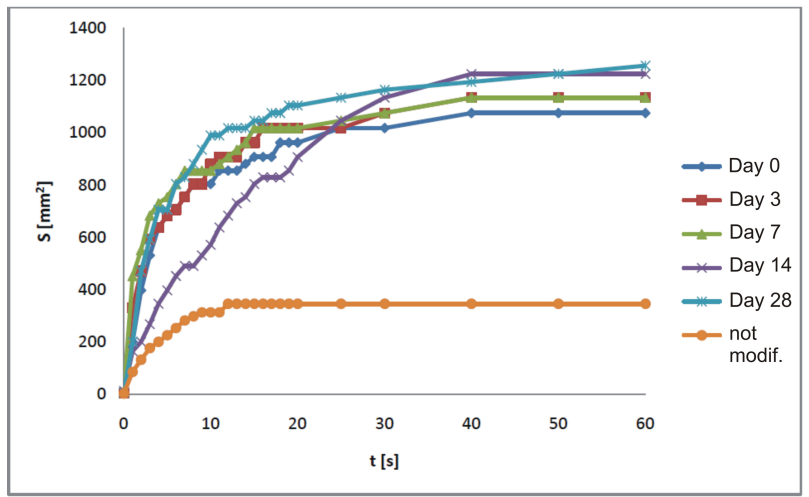

FIGURE 4. E-glass textile modified sample, wettability expressed by a print area, modification time $10 \mathrm{~s}$.

tile samples made from E-glass a considerable effect of the wettability change was observed after the exposure in plasma, as one can see in the attached diagrams (Figs. 2 44). The modified textile samples were stored at room temperature and atmospheric pressure in a dark place in a container protecting the samples against dust and enabling access of humidity.

The increase of wettability was apparent immediately after the modification and it is obvious from the diagrams that the imprint of the drop with potassium permanganate solution is three times larger after 28 days in comparison with the sample without any modification.

The wettability increase during ageing is a very interesting result of the test. It was probably caused by the subsequent reactions on the modified fibre surface. From comparison of the behaviour of the samples stored in a desiccator without access to ambient humidity and in open atmosphere (air with natural humidity), one can derive that radicals created on the sample surface during the plasma modification react with the air humidity from the open atmosphere. These formed radicals cause hydrophilic behaviour of the sample surface. The ageing effect (enhancement of the hydrophilicity) was not observed in such an extent if the samples were stored in a desiccator without access to ambient humidity. Without access to ambient humidity, where e.g. the samples aged 28 days reached the print area size 1.5 larger only in case of $10 \mathrm{~s}$ modification in comparison with the sample without any modification.

The positive effect of the modification ageing was registered only for the samples that were stored in the environment with humidity. The effect of the wettability increase was very significant in this way of storage in comparison with the initial level of wettability of the samples without modification (Fig. 7). However there are certain differences among the samples with different times of plasma treatment (exposure). The samples with the modification time of $1 \mathrm{~s}$ and $5 \mathrm{~s}$ have a slowly upward curve of wettability in comparison with the samples modified for $10 \mathrm{~s}$ (Figs. 577).

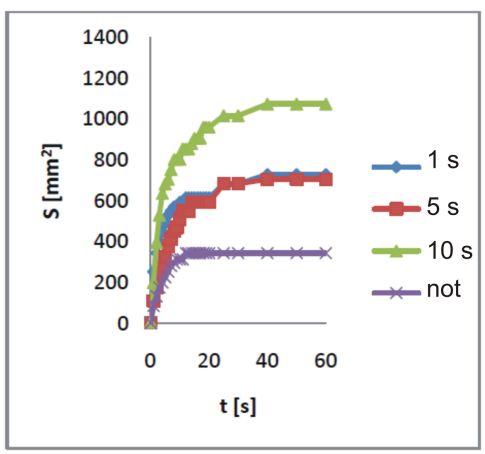

Figure 5. Ageing effect on an E-glass textile modified sample, Day 0, not modified sample displayed for comparison.

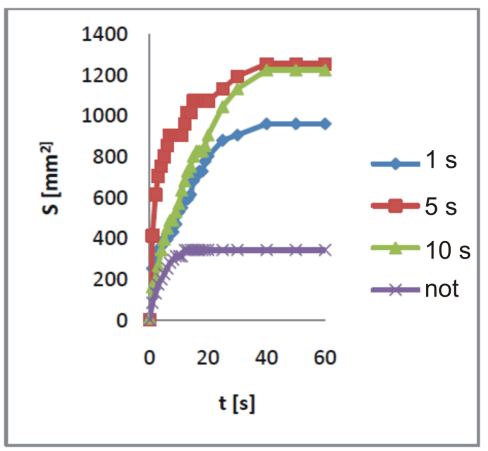

Figure 6. Ageing effect on an E-glass textile modified sample, Day 14, not modified sample displayed for comparison.

\subsection{SEM ANALYSIS OF MODIFIED TEXTILES}

A visual check of all samples did not show any macroscopic damages such as for example holes in the samples caused by the barrier discharge. The detailed analysis of the samples by means of SEM method was carried out with the objective to determine possible small damages of the samples caused by the exposure to plasma. The SEM analysis did not prove any destroyed or damaged fibres because of exposure to DBD plasma. There are no visible differences between the samples before and after the plasma modification (Figs. 8 9). This is a very important fact from the standpoint of a possible future application of this method for the fibres used as fillers for composite materials.

Only small particles are visible on the fibres (Fig. 9) that probably contaminated the textile samples in the form of dust, which was caught electrostatically during handling and analyses.

\section{Conclusion}

The interaction of the dielectric barrier discharge and fibre materials used as fillers in the composite materials seems to be a good topic for further research focused on an increase of their wettability and adhesion to the matrix. The modification effectiveness is sufficient so we can evaluate its positive effect even by means of simple measuring methods and we can 


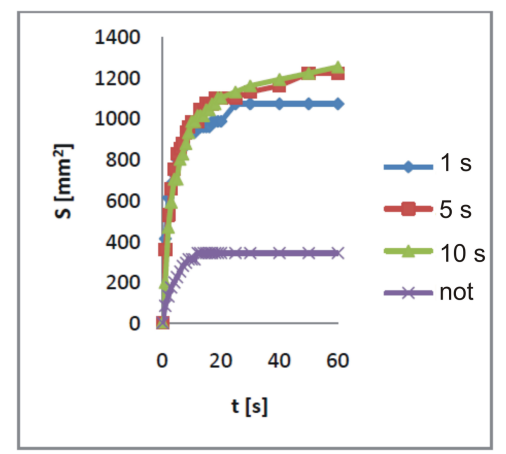

Figure 7. Ageing effect on an E-glass textile modified sample, Day 28, not modified sample displayed for comparison.

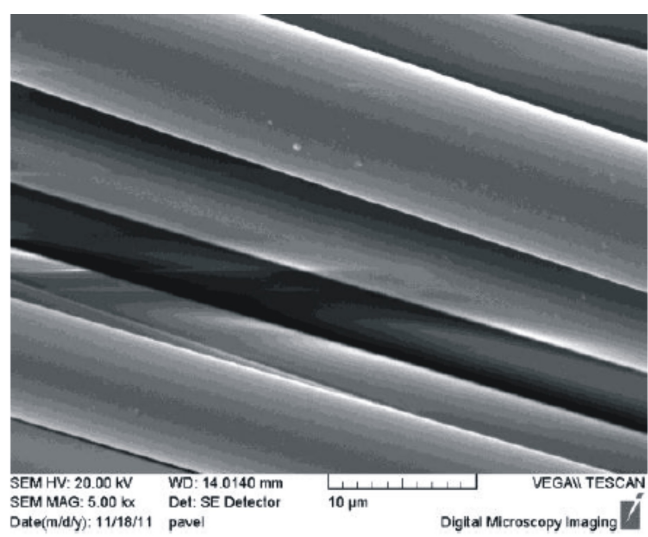

Figure 8. SEM image of an E-glass textile, not modified sample.

also study the modification ageing. Many more samples have to be modified and tested in order to identify optimum conditions for the increasing of interfacial bonding strength caused by the wettability increase. The result evaluation has proved the positive behaviour of the tested textiles immediately after the plasma modification as well as during the following weeks, when the continuing effect of the surface modification was observed.

\section{ACKnowledgements}

We would like to thank to the Technological centre of company MSV SYSTEMS CZ s.r.o. for their help with the construction and building of DBD plasma device and bringing it into the operation.

\section{REFERENCES}

[1] T. C. Chang. Plasma surface treatment in composites manufacturing. Journal of Industrial Technology 15(1), 1999.

[2] C. Cheng, Z. Liye, R-J. Zhan. Surface modification of polymer fibre by the new atmospheric pressure cold plasma jet. Surface $\&$ Coatings Technology 200:6659-6665, 2006.

[3] F. Ferrero. Wettability measurements on plasma treated synthetic fabrics by capillary rise method. Polymer Testing 22:571-578, 2003.

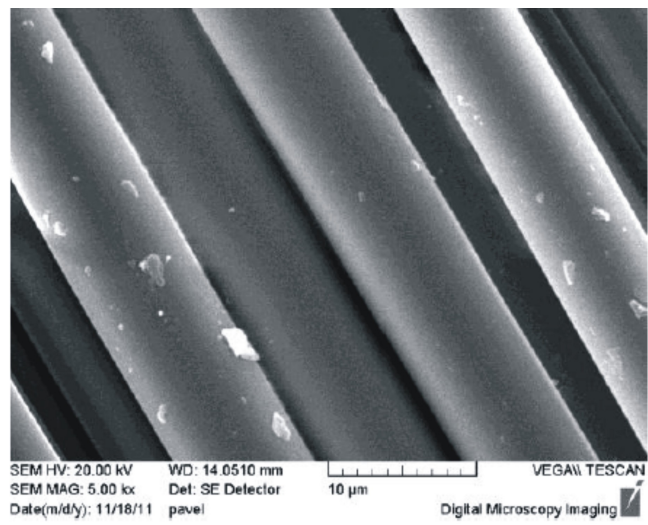

Figure 9. SEM image of an E-glass textile, 10s DBD modified sample.

[4] Y. Klenko, J. Píchal, L. Aubrecht. Atmospheric DBD discharge modication of polyester fabric. Problems of Atomic Science and Technology, Series Plasma Physics 12(6):195-196, 2006.

[5] J. Píchal, D. Hollá, J. Sláma. Treatment of polyester fabric in atmospheric dielectric barrier discharger operated at low frequencies. Acta Technica CSAV 56(supp.):T223-T227, 2011.

[6] J. Píchal, J. Sláma. Polyester fabric modified with atmospheric dielectric barrier discharge supplied with voltage of different frequencies. Problems of Atomic Science and Technology 16(6):188-190, 2010.

[7] H. U. Poll, U. Schladitz, S. Schreiter. Penetration of plasma effects into textile structures. Surface Coating Technology 142-144:489-493, 2001.

[8] Y. Ren, C. Wang, Y. Qiu. Aging of surface properties of ultra high modulus polyethylene fibers treated with $\mathrm{He} / \mathrm{O} 2$ atmospheric pressure plasma jet. Surface 85 Coatings Technology 202:2670-2676, 2008.

[9] Yu Ren, Chunxia Wang, Yiping Qiu. Influence of aramid fiber moisture regain during atmospheric plasma treatment on ageing of treatment effects on surface wettability and bonding strength to epoxy. Applied Surface Science 253:9283-9289, 2007.

[10] C. Tendero, C. Tixier, P. Tristant, et al. Atmospheric pressure plasmas: A review. Spectrochimica Acta Part B 61:2-30, 2006.

[11] J. M. Thiebaut, T. Belmonte, D. Chaleix, et al. Comparison of surface cleaning by two atmospheric pressure discharges. Surf Coat Technol 169-170:181-185, 2003.

[12] C. M. Weikart, M. Miyama, H. K. Yasuda. Surface modification of conventional polymers by depositing plasma polymers of trimethylsilane and trimetylsilane + O2: I. Static wetting properties. J Colloid Interface Sci 211(1):18-27, 1999. 